\title{
Kernos
}

Revue internationale et pluridisciplinaire de religion grecque antique

$25 \mid 2012$

Varia

\section{Angelos CHANIOTIS (éd.), Ritual Dynamics in the Ancient Mediterranean. Agency, Emotion, Gender, Representation}

\section{Aurian Delli Pizzi}

\section{(2) OpenEdition}

\section{Journals}

Édition électronique

URL : http://journals.openedition.org/kernos/2073

DOI : 10.4000/kernos.2073

ISSN : 2034-7871

\section{Éditeur}

Centre international d'étude de la religion grecque antique

\section{Édition imprimée}

Date de publication : 26 octobre 2012

Pagination : 369-372

ISSN : 0776-3824

Référence électronique

Aurian Delli Pizzi, «Angelos chaniotis (éd.), Ritual Dynamics in the Ancient Mediterranean. Agency, Emotion, Gender, Representation », Kernos [En ligne], 25 | 2012, mis en ligne le 01 octobre 2012, consulté le 21 septembre 2020. URL : http://journals.openedition.org/kernos/2073 ; DOI : https:// doi.org/10.4000/kernos.2073

Ce document a été généré automatiquement le 21 septembre 2020 


\title{
Angelos CHANiotis (éd.), Ritual Dynamics in the Ancient Mediterranean. Agency, Emotion, Gender, Representation
}

\author{
Aurian Delli Pizzi
}

\section{RÉFÉRENCE}

Angelos CHANiоTis (éd.), Ritual Dynamics in the Ancient Mediterranean. Agency, Emotion, Gender, Representation, Stuttgart, Franz Steiner Verlag, 2011. 1 vol. $17 \times 24$ cm, 390 p. (Heidelberger Althistorische Beiträge und Epigraphische Studien, 49). ISBN :

978-3-515-09916-5.

1 Cet ouvrage se penche sur la question des transformations à l'œuvre dans les traditions rituelles des religions méditerranéennes antiques, avec une attention particulière portée aux acteurs à l'origine de ces changements ainsi qu'aux émotions suscitées. Plusieurs contributions sont issues de communications présentées dans le panel "agency " du colloque "Ritual Dynamics and the Science of Ritual », organisé à Heidelberg en 2008. Je résume ici les contributions portant sur le monde grec.

L'antithèse tradition-innovation est un des fils conducteurs de l'ouvrage, mais l'accent est mis, à juste titre, sur les moyens de conciliation entre ces deux éléments plutôt que sur leur incompatibilité. L'article de E. Stavrianopoulou porte sur les conséquences de la mise par écrit de la tradition. Comme le suggèrent les poursuites engagées contre Nicomachos à la suite de son activité d'anagrapheus des lois concernant les sacrifices, la mise par écrit des sacrifices qui doivent être accomplis met en lumière, aux yeux des Grecs, certains changements par rapport à ce qui est conçu, de manière assez vague, comme la "tradition ». Par ailleurs, le décret qui fait état de la restauration des Thargélies à Athènes en 129/8 av. J.-C (LSS 14) évoque, à plusieurs reprises, les origines 
de cette fête en vue de justifier la restauration de celle-ci. Dans ce cas-ci, ce sont précisément les changements qui élaborent, a posteriori, la tradition. En définitive, la mise par écrit d'éléments rituels conformément à la tradition ne vise pas à suivre scrupuleusement des prescriptions ancestrales, mais à figer une version de la tradition telle qu'elle est interprétée et modifiée dans un contexte précis, et à servir de fondement pour des changements ultérieurs. Différents types de changements à l'œuvre dans les mystères d'Éleusis sont étudiés dans l'article de I. Patera. Le calendrier de l'initiation aux Mystères pouvait être modifié en raison de facteurs politiques ou religieux. Le rituel pouvait être abandonné dans sa totalité, comme ce fut le cas en 335 av. J.-C., à la suite de la destruction de Thèbes par Alexandre, ou en partie, comme lorsque la procession ne fut pas organisée lors de la guerre du Péloponnèse. Parallèlement, le rôle joué par Athènes dans la désignation du personnel d'Éleusis n'a fait qu'augmenter: à titre d'exemple, dans le décret portant sur la dédicace des prémices (IG I ${ }^{3} 78$ ), le rôle des hieropoioi s'accroît au détriment des administrateurs traditionnels. Enfin, des modifications apportées au rituel transparaissent dans les sources archéologiques (constructions successives de bûchers, nombreux kernoi et plemochoai).

3 Par ailleurs, les rôles respectifs d'un individu ou d'une communauté entière dans la modification d'un rituel sont mis en exergue. P. Martzavou étudie le rôle des différents agents qui interviennent dans le culte d'Isis en Grèce, qu'il s'agisse de prêtres ou de particuliers. Elle insiste sur le rôle de Délos dans la diffusion du culte isiaque en Grèce et décrit l'implication d'Athènes dans le recrutement des prêtres d'Isis sur cette île. Elle se concentre sur les reliefs funéraires athéniens qui présentent la figure d'Isis Dikaiosunè, probablement importée de Délos et accompagnée de différents attributs isiaques (sistre, vase, nœud isiaque). Bien que, selon elle, l'initiation à Isis relève davantage, à la différence des mystères d'Éleusis, d'une démarche personnelle, elle note une série de points communs entre les deux types d'initiation (présence de canéphores, parallèles épigraphiques et rituels). À partir de ces différents éléments, elle avance l'hypothèse d'un processus de "sacerdotisation", par le biais duquel des individus initiés au culte d'Isis agiraient comme des prêtres «freelance ». F. Graf présente trois cas d'innovations cultuelles dans deux cités grecques de l'époque impériale, Éphèse et Akraiphia. Tout d'abord, en 44 ap. J.-C., dans le cadre du culte éphésien d'Artémis, le gouverneur romain Paullus Fabius Maximus remplaça les hymnodoi professionnels par des éphèbes. Même si cette démarche constitue un retour à une tradition antérieure, l'argument principal est de réduire le budget nécessaire. En 104 ap. J.-C., C. Vibius Salutarius fit don d'une importante somme d'argent à la cité d'Éphèse, principalement pour financer l'érection de statues qui représentaient Artémis ainsi que des institutions romaines et éphésiennes. En outre, la restauration de la fête consacrée à Apollon Ptoios dans la cité béotienne d'Akraiphia par Épaminondas, qui venait de remplir une mission d'ambassadeur auprès des Romains, montre comment il était possible de concilier élite locale et pouvoir romain. En revanche, l'analyse de S. des Bouvrie indique que, dans le cas du théâtre attique, ce n'est pas l'agency d'un individu qui est à la manœuvre, mais bien une collectivité. Après une introduction théorique fouillée où elle pose la question de savoir si le théâtre antique doit être considéré comme un rituel, elle décrit le déroulement des Dionysies afin de mettre en exergue les différentes phases émotionnelles par lesquelles passaient les participants de cette fête (cf. notamment l'importance de la raillerie, tôthasmos, dans la mise en place d'une atmosphère émotionnelle particulière). Elle se fonde également sur des développements politico- 
historiques pour montrer comment les rapports entre masse et élite conditionnaient le déroulement de ces manifestations.

4 Les évolutions que connaissent les rites funéraires, ainsi que les tensions entre prescriptions de la cité et démarches personnelles qui en découlent, font l'objet de l'article de F. Frisone. Il y est question de la place des règlements funéraires au sein des dites «lois sacrées» et de la nécessité de concevoir ces textes comme règlements rituels plus que comme des lois somptuaires. Par ailleurs, les rituels funéraires constituent une manière de s'insérer ou non dans un canevas de valeurs définies par la communauté. Tout en montrant comment certains de ces règlements pouvaient être modifiés à des fins politiques (cf. décret de Thasos sur l'interdiction d'être en deuil pour les combattants défunts pour la patrie), F. Frisone réfléchit également à la place des femmes dans ces règlements.

5 A. Chaniotis étudie la manière dont certaines émotions, comme la peur ou l'espoir, apparaissent dans le cadre de la communication entre une communauté et un dieu, dans différents contextes spécifiques. Il attire notamment l'attention sur le fait que les communautés impliquées dans des cultes initiatiques sont particulièrement fondées sur des émotions. Par ailleurs, la relation entre la cité d'Éphèse et sa déesse Artémis est un cas particulier : Artémis n'est pas uniquement la divinité « tutélaire » de cette cité, mais un véritable rapport d'affection s'établit entre la déesse et les habitants d'Éphèse. Enfin, les pèlerins qui se rendent au sanctuaire de la Mère des dieux de Leukopétra et font inscrire sur pierre les consécrations d'êtres humains offrent un autre cas d'étude. Selon A. Chaniotis, ces émotions partagées dans un cadre cultuel précis ont pour résultat que certaines communautés cultuelles deviennent des communautés émotionnelles. La question des émotions atteint son apogée dans l'article original de J.B. Connelly, qui étudie le mouvement du corps humain dans l'espace comme acte rituel, en interaction avec les bâtiments qui entourent les individus à l'œuvre. Le premier cas d'étude porte sur les théories athéniennes (en réalité, l'A. classe également dans cet en-tête une série de processions liées à des fêtes locales). Il est également question des courses de jeunes filles, organisées notamment dans le sanctuaire d'Artémis à Messène, ainsi que de la danse des jeunes gens et des jeunes filles lors de la pannychis sur le flanc de l'acropole athénienne. J.B. Connelly suggère en outre de comprendre une plateforme circulaire mise au jour sur l'îlot de Yéronisos, à l'ouest de Chypre, comme une piste de danse. Cet article permet d'entrevoir comment certaines pratiques rituelles s'inséraient dans des infrastructures construites ou naturelles, à condition de prendre avec précaution certaines hypothèses qui y sont avancées de manière assez spéculative.

6 En comparaison des autres contributions décrites ci-dessus, l'article de F. Létoublon s'éloigne quelque peu des émotions et du concept d'agency. L'A. pose en effet la question de savoir si les formules codifiées des poésies homérique et hésiodique correspondent à des formules rituelles, ou si elles ont une dimension purement littéraire. Elle étudie d'abord les scènes de prière, contrastant les prières solennelles

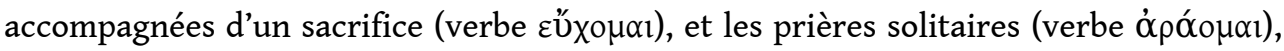
en reconnaissant d'emblée les limites d'une telle distinction. Il y a fort à parier que tout le monde ne suivra pas son refus d'attribuer aux imprécations toute valeur d'acte langagier. Elle met bien en exergue que les ressemblances entre différentes scènes «typiques » se justifient notamment en raison de l'économie de l'œuvre-même : dans le cas des scènes de supplication, notamment, les paroles prononcées par les agents de 
l'histoire épargnent au narrateur le besoin de décrire le procédé de supplication par des détails minutieux.

7 Bien que la majorité des articles concernent le monde grec, il serait injuste de passer sous silence les contributions qui portent sur d'autres lieux. E. Perego s'intéresse au rôle des femmes dans les rituels de la Vénétie pré-romaine. M. López-Bertran étudie des figurines d'Illa Plana (îles Baléares) pour illustrer les pratiques rituelles locales. M.M. McCarty traite du changement de la conception du sacrifice dans le monde punique lors des contacts avec les Romains. A. Verbovsek livre un article très intéressant sur les rapports entre émotions et rituels en Égypte. Enfin, M. Icks traite des cérémonies d'investiture impériales romaines, dont les irrégularités étaient parfois présentées comme annonciatrices du mauvais règne d'un empereur. Même si le sujet de ces articles intéresse moins directement les hellénistes, leurs développements théoriques, ainsi que les applications qu'ils proposent, valent la peine d'être pris en compte dans le cadre d'une réflexion sur la religion grecque.

\section{AUTEURS}

AURIAN DELLI PIZZI

Université de Liège 